\title{
No Association Between Markers of Inflammation and Osteoarthritis of the Hands and Knees
}

\author{
STEVEN C. VLAD, TUHINA NEOGI, PIRAN ALIABADI, JOÃO D.T. FONTES, and DAVID T. FELSON
}

ABSTRACT. Objective. Local inflammation plays a prominent role in osteoarthritis (OA). This could be reflected in the presence of elevated soluble inflammatory markers. We conducted analyses to assess the association of inflammatory markers with radiographic OA of the hands and knees in a large community-based cohort.

Methods. The Framingham Offspring cohort consists of the adult children of the original cohort and their spouses. In 1998-2001 these subjects provided blood specimens that were tested for 17 markers of systemic inflammation. In 2002-2005 these subjects had radiographs of both knees and hands. Each hand and knee joint was assigned a Kellgren and Lawrence (KL) score (0-4). We used logistic regression with generalized estimating equations and adjustment for age, sex, and body mass index to examine the association between each inflammatory marker and the presence of radiographic OA $(\mathrm{ROA}=$ KL grade $\geq 2$ ) in any joint. We also constructed models for hand joints and knee joints alone.

Results. Radiographs and measures of inflammation were done for 1235 subjects (56\% women, mean age $65 \mathrm{yrs})$. Of that group, 729 subjects (59\%) had ROA in $\geq 1$ hand or knee joint: 179 (14.3\%) had knee OA, and $694(56.2 \%)$ had hand OA. There were no significant associations between any marker of inflammation and ROA.

Conclusion. In this large sample, in which OA was carefully assessed and multiple markers measured, we found no evidence of an association between any inflammatory marker and the presence of radiographic OA. (First Release May 15 2011; J Rheumatol 2011;38:1665-70; doi:10.3899/jrheum.100971)

Key Indexing Terms: OSTEOARTHRITIS

BIOLOGICAL MARKERS

INFLAMMATION

Osteoarthritis (OA), the prototypical "noninflammatory" arthropathy, is now well recognized to involve an inflammatory component ${ }^{1}$. Inflammatory cytokines produced by the synovium and chondrocytes, including interleukin $1 \beta$ (IL-1ß), tumor necrosis factor- $\alpha$ (TNF- $\alpha$ ), IL- 6 , and others, appear to play pivotal roles in cartilage destruction. These cytokines are produced by the synovium and chondrocytes and are expressed there and in the synovial fluid ${ }^{2,3}$.

From the Clinical Epidemiology Research and Training Unit and the Division of Rheumatology, Boston University School of Medicine; Department of Radiology, Brigham and Women's Hospital; Cardiology Division, Boston University School of Medicine, Boston; the National Heart, Lung, and Blood Institute's Framingham Heart Study,

Framingham, Massachusetts, USA.

Supported by NIH grants T32 007598, K23 AR056301, K23 AR055127, R01 HL076784, R01 AG028321, R01 HL064753, R01 AG018393, P60 AR047785, and N01 HC025195.

S.C. Vlad, MD, Assistant Professor of Medicine; T. Neogi, MD, PhD, Associate Professor of Medicine, Clinical Epidemiology Research and Training Unit and Division of Rheumatology, Boston University School of Medicine; P. Aliabadi, MD, Department of Radiology, Brigham and Women's Hospital; J.D.T. Fontes, MD, Cardiology Division, Boston University School of Medicine, and the National Heart, Lung, and Blood Institute's Framingham Heart Study; D.T. Felson, MD, MPH, Professor of Medicine, Clinical Epidemiology Research and Training Unit and Division of Rheumatology, Boston University School of Medicine. Address correspondence to Dr. S.C. Vlad, Boston University School of Medicine, Clinical Epidemiology Research and Training Unit, 650 Albany Street, Suite X200, Boston, MA 02118, USA.E-mail: svlad@bu.edu Accepted for publication March 8, 2011.
Some studies suggest that this local inflammation may be reflected systemically. This has been best studied with high-sensitivity C-reactive protein (CRP) serving as a marker for systemic inflammation, with several studies showing a relationship between serum CRP levels and OA of the knee $^{4,5,6}$, hip ${ }^{6,7}$, and hands ${ }^{8}$. Others have found serum CRP to reflect local evidence of joint inflammation ${ }^{9}$ or pain associated with $\mathrm{OA}^{10}$. Other studies, however, have reported no association of CRP with OA, especially after adjustment for the influence of body mass ${ }^{10,11,12,13}$.

It is possible that CRP is not an optimal marker for inflammation in $\mathrm{OA}$; other putative markers have been associated with some OA phenotypes. These include IL- $6^{14,15}$, TNF- $\alpha^{15}$, TNF-receptor II (TNF-RII) ${ }^{14}$, IL-1 ${ }^{16}$, intercellular adhesion molecule 1 (ICAM-1) ${ }^{17}$, and adiponectin ${ }^{18}$.

Given the local role of inflammatory cytokines in OA and the potential trafficking of inflammatory cells through OA synovium and back out into the circulation ${ }^{2}$, there is reason to believe that some systemic inflammatory markers may be present at higher or lower than normal levels in the blood of subjects with OA, and it is possible that some of these may correlate with specific OA phenotypes. An absence of associations might suggest that inflammation is a very localized phenomenon without significant systemic effects. We examined whether elevations in some inflammatory markers known to be associated with OA, and others not yet clearly

Personal non-commercial use only. The Journal of Rheumatology Copyright @ 2011 . All rights reserved. 
associated with $\mathrm{OA}$, were associated with radiographic OA (ROA) of the hands and/or knees in a large population-based cohort. We hypothesized that some of these markers (e.g., CRP) would be elevated in those with hand and/or knee OA.

\section{MATERIALS AND METHODS}

We used data from the Framingham Offspring Cohort. This community-based cohort consists of the adult children of members of the original Framingham Heart Study, as well as the spouses of these children. The study began in 1971 and has now completed 8 examination cycles, which take place every 4 years. Details of the selection and design have been described ${ }^{19}$.

During the seventh examination cycle (1998-2001), as part of a study of inflammatory markers and heart disease, blood samples were collected from subjects. Standardized assays were used to measure serum markers of inflammation that could be associated with coronary heart disease; we examined their possible relevance to OA. These markers included adiponectin, plasma CD40 ligand, serum CD40 ligand, CRP, fibrinogen, ICAM-1, IL-6, lipoprotein-associated phospholipase $\mathrm{A}_{2}$ (LP-PLA ${ }_{2}$ ) activity and mass, monocyte chemoattractant protein 1 (MCP-1), myeloperoxidase (MPO), osteoprotegerin (OPG), P-selectin, resistin, TNF- $\alpha$, and TNF-RII. Urine was also collected and assays were conducted to measure urine isoprostane levels corrected for the level of urine creatinine. In the case of outliers, sources of laboratory error were investigated and if none were found, the outlier was retained in the data. Erroneous values, values below the detectable limits of the assays, or missing data due to inadequate sample size were characterized as missing. Details of the assays, including intraassay coefficients of variation, have been published ${ }^{20}$.

In 1992-95, a subset of the Framingham Offspring cohort was invited to participate in a study of OA, the Framingham Osteoarthritis Study. At callback examinations from 2002 to 2005, a sample of 1235 of these participants underwent standardized, weight-bearing, fixed-flexion radiographs of the knees and standardized posteroanterior radiographs of the hands. These protocols have been described ${ }^{21,22}$. Subjects with a history of inflammatory joint disease were excluded. All radiographs were read by a single academically based musculoskeletal radiologist and each hand and knee joint was assigned a Kellgren and Lawrence $(\mathrm{KL})$ score from 0 to 4: $0=$ no osteophytes or joint space narrowing, 1 = possible osteophytes, $2=$ definite osteophytes and possible joint space narrowing, $3=$ moderate osteophytes and definite joint space narrowing, and $4=$ large osteophytes, severe joint space narrowing, and/or bony sclero$\operatorname{sis}^{23}$. The intraobserver weighted $\kappa$ were 0.77 for hand KL grade, and 0.91 for knee $\mathrm{KL}$ grade. We defined the presence of ROA as any KL score $\geq 2$.

Age and body mass index (BMI) were obtained at the same visits at which radiographs were obtained.

Analysis. All inflammatory marker measurements were standardized by dividing values by the respective SD for the relevant assay so as to allow comparisons between different markers. Therefore, OR represents the increase in the odds of ROA for a 1 SD increase in the inflammatory marker. A number of models were constructed to assess the relationship between an inflammatory marker and ROA. In each case crude models and those adjusted for age, sex, and BMI were constructed. Interaction between each marker and age, sex, and BMI were explored; the number of potential interactions found were consistent with chance, given the number of tests performed, and thus are not reported. Therefore the models without interaction are reported.

The first set of models used a joint-centered definition of ROA. We used logistic regression with generalized estimating equations (GEE) to account for multiple joints within a subject. Models were created with the inflammatory marker as the independent variable and the presence of ROA in any joint (knees or hands) as the dependent variable. The models were also repeated using only hand or only knee joints as the dependent variables.

We constructed a second set of models using subject-centered definitions of ROA to find evidence of associations between the inflammatory markers and specific, more severe OA phenotypes, where inflammation might be more likely to play a role. Again using logistic regression (without GEE), the inflammatory marker was the independent variable, while "presence of OA" in a subject was the dependent variable. In these models, we tested several different definitions of "presence of OA": (1) the presence of ROA in both thumb bases; (2) the presence of ROA in 4 or more distal interphalangeal (DIP) joints (including the first IP joint) of each hand; (3) the presence of ROA in 4 or more proximal interphalangeal (PIP) joints (including the first IP joint) of each hand; (4) the presence of ROA in 4 or more metacarpophalangeal (MCP) joints of each hand; and (5) the presence of ROA in both knees.

For a third set of models, we used the total joint load, i.e., the number of joints affected, as the outcome variable. For these models, we used linear regression with the total number of affected joints as the outcome variable. As the number of joints affected was not normally distributed, we repeated the analysis using the natural logarithm of the number of joints as the outcome. We also modeled higher-ordered relationships (square of the marker and square root of the marker).

We then explored the possibility that OA could be the cause of changes in inflammatory marker levels. We created linear models where the total number of joints affected by ROA served as the independent variable and the logarithm of the standardized inflammatory marker served as the outcome variable. The B-coefficients in these models reflect the expected increase in the marker (in standard deviations) for each additional joint affected by ROA. Models were created with and without adjustment for age, sex, and BMI. Linear models alone were thought to be sufficient as visual inspection of the primary data (logarithm of marker vs total number of joints) and residual plots did not suggest nonlinear relationships. Plots and analyses using LOESS regression also did not suggest more complex relationships.

Finally, as inflammation could be more strongly associated with painful OA rather than ROA, we performed sensitivity analyses in which we redefined the "presence of OA" to require pain in the affected hand or knee, as well as radiographic evidence of $\mathrm{OA}(\mathrm{KL} \geq 2)$.

\section{RESULTS}

The study sample consisted of 1235 people, of whom 689 $(56 \%)$ were women (Table 1). The mean age was 65.3 years (SD 9.1, median 65); 897 subjects (73\%) were age 60 years or older. The mean BMI was 28.6 (SD 5.5, median 28.0). Not all subjects had assays for each inflammatory marker.

There were 729 subjects (59\%) who met our definition of ROA in $\geq 1$ joint: 413 women (60\%) and 316 men (58\%). Using our definitions, 317 subjects (26\%) had thumb base OA, 76 (6\%) had DIP joint OA, 28 (2\%) had PIP OA, and 15 $(1 \%)$ had MCP OA. There were $89(7 \%)$ with bilateral knee

Table 1. Demographic data of the cohort and details of radiographic osteoarthritis (ROA) cases.

\begin{tabular}{lc}
\hline Characteristics & Mean (SD) or n (\%) \\
\hline Age, yrs & $65.3( \pm 9.1)$ \\
Body mass index & $28.6( \pm 5.5)$ \\
Women & $689(56)$ \\
Any radiographic OA (KL $>2$ in any joint) & $729(59)$ \\
Men & $316(58)$ \\
Women & $413(60)$ \\
Thumb base OA (KL $\geq 2$ in both hands) & $317(26)$ \\
DIP OA (KL $\geq 2$ in 4 DIP joints of each hand) & $76(6)$ \\
PIP OA (KL $\geq 2$ in 4 PIP joints of each hand) & $28(2)$ \\
MCP OA $(K L \geq 2$ in 4 MCP joints of each hand) & $15(1)$ \\
Knee OA $(K L \geq 2$ in both knees) & $89(7)$ \\
\hline
\end{tabular}

KL: Kellgren and Lawrence grade; DIP: distal interphalangeal; PIP: proximal interphalangeal; MCP: metacarpophalangeal. 
OA. In measuring pain, we found that 114 subjects $(9 . \%)$ had painful thumb base OA, 44 (4\%) had painful DIP OA, $16(1 \%)$ had painful PIP OA, and $3(0.2 \%)$ had painful MCP OA. Forty-six (4\%) had painful bilateral knee OA.

The joint-centered logistic regression models failed to demonstrate convincing associations between any of the inflammatory markers and ROA (Table 2). A marginal association was seen for serum CD40.

The patient-centered models also failed to find any convincing association between any of the markers and our ROA definitions (Table 3). Although some statistically significant associations were observed, these tended to be small, with 95\% CI barely excluding unity. Other associations showed contradictory effects; for example, IL-6 was associated with both a higher and lower risk of OA, depending on the OA phenotype assessed. Of note, CRP was associated with a lower risk of OA in the DIP joints.

Neither linear regression model revealed an association between any marker and the total "load" of ROA (Table 4). Higher-order models suggested that 5 markers might have a relationship with the joint load. Four of these were of questionable significance [square root of TNF-RII $(\beta$-coefficient $=$ $0.28, \mathrm{p}=0.06)$; OPG squared ( $($-coefficient $=0.07, \mathrm{p}=0.04)$; square root of MPO (B-coefficient $=0.30, p=0.04)$; and LP-PLA $_{2}$ mass squared ( $($-coefficient $\left.=-0.015, \mathrm{p}=0.04)\right]$. Another marker (serum CD40) was slightly more suggestive, with the $\beta$-coefficient for the square root $=0.30, p=0.03$. However, given the large number of comparisons, none of

Table 2. Joint-specific OR of radiographic osteoarthritis (ROA) for each marker. OR data are of OA in any joint, using logistic regression and accounting for multiple correlations between joints with generalized estimating equations; adjusted for age, sex, and body mass index.

\begin{tabular}{lcc}
\hline Marker & $\begin{array}{c}\text { N in Analysis } \\
\text { (Maximum n = 1235) }\end{array}$ & $\begin{array}{c}\text { OR of ROA for 1 SD } \\
\text { Increase of Marker (95\% CI) }\end{array}$ \\
\hline Adiponectin & 885 & $1.02(0.91-1.14)$ \\
CD40 (serum) & 1211 & $1.10(1.00-1.18)$ \\
CD40 (plasma) & 1229 & $0.99(0.91-1.08)$ \\
C-reactive protein & 1226 & $0.98(0.89-1.07)$ \\
Fibrinogen & 1227 & $0.94(0.85-1.03)$ \\
ICAM-1 & 1226 & $1.00(0.92-1.08)$ \\
Interleukin 6 & 1225 & $0.97(0.89-1.05)$ \\
Isoprostanes & 964 & $1.01(0.91-1.13)$ \\
LP-PLA ${ }_{2}$ activity & 1227 & $0.95(0.86-1.06)$ \\
LP-PLA 2 mass & 1227 & $0.95(0.87-1.04)$ \\
MCP1 & 1208 & $1.01(0.95-1.07)$ \\
Myeloperoxidase & 1182 & $1.06(0.99-1.15)$ \\
Osteoprotegerin & 1227 & $0.99(0.90-1.09)$ \\
P-selectin & 1229 & $0.97(0.89-1.05)$ \\
Resistin & 876 & $0.98(0.88-1.08)$ \\
TNF- $\alpha$ & 877 & $0.97(0.91-1.04)$ \\
TNF-RII & 1194 & $1.04(0.95-1.13)$ \\
\hline
\end{tabular}

CD40: CD40 ligand; ICAM-1: intercellular adhesion molecule 1; isoprostanes: (urinary creatinine)/(8epi-PGF2a); LP-PLA 2 : lipoprotein-associated phospholipase $\mathrm{A}_{2}$; $\mathrm{MCP} 1$ : monocyte chemoattractant protein; TNF- $\alpha$ : tumor necrosis factor- $\alpha$; TNF-RII: type II TNF receptor. these relationships can be said to show a definitive relationship with the total joint load.

Linear regression models in which the affected joint count served as the independent variable and the logarithm of the standardized marker level served as the outcome also failed to show any convincing relationship (Table 5). Although some nominally statistically significant outcomes occurred, these effects were small, and given the number of associations tested, likely occurred by chance.

In the sensitivity analysis, using symptomatic $\mathrm{OA}$ as the primary outcome, we again generally failed to find concrete evidence of a relationship between any inflammatory marker and OA (results not shown). However, relatively strong associations of potential interest include fibrinogen with DIP OA (OR 0.65, 95\% CI 0.45, 0.95); IL-6 with DIP OA (OR 0.31, 95\% CI 0.12, 0.77); LP-PLA 2 activity with PIP OA (OR 1.8, 95\% CI 1.1, 3.0); and LP-PLA 2 mass with PIP OA (OR 2.0, $95 \%$ CI $1.3,3.1)$.

\section{DISCUSSION}

We failed to find convincing evidence of a cross-sectional relationship between any systemic inflammatory marker and ROA. Despite a number of studies suggesting a relationship between serum CRP levels and OA, we could not reproduce this finding in this community-based sample. Nor did we demonstrate a convincing link between any other marker of inflammation and the radiographic phenotypes we defined.

Studies of the link between OA and CRP, the most-studied potential biomarker of OA, have had contradictory findings. In general, large population-based studies with adequate control for body mass have failed to find a convincing association $^{12,13,24}$, while those of selected OA populations, often using healthy controls, have found an association $4,7,8,14,25,26$, although exceptions are found in both cases ${ }^{5,11}$. This suggests that at least some of the latter studies may suffer from residual confounding due to insurmountable differences between cases and controls. The findings from our study with regard to CRP would be consistent with this hypothesis.

However, 2 studies deserve special mention in this regard: Sowers and colleagues ${ }^{27}$ in their population-based study suggested that, although the association of CRP with OA was greatly attenuated when adjusted for body mass, there could be remaining effect modification by CRP of the weight-OA association. (We were unable to find effect modification of any inflammatory marker by BMI.) Bos and colleagues ${ }^{28}$ investigated the association of CRP haplotypes and both serum CRP levels and the development of OA. Although 1 haplotype (H7/8) was associated with both serum CRP and OA, serum levels of CRP were not associated with OA. Another recent report ${ }^{24}$ found no association between CRP haplotype and OA. CRP haplotype in Bos and colleagues ${ }^{28}$ appears to have served as an instrumental variable (Mendelian randomization), allowing the authors to detect a relationship with OA, while the serum level was too confounded to

Personal non-commercial use only. The Journal of Rheumatology Copyright @ 2011. All rights reserved. 
Table 3. OR of radiographic osteoarthritis (ROA) using patient-centered definitions. OR data are for each definition of OA, using logistic regression and adjusted for age, sex, and BMI. Bold type indicates statistically significant associations $(\mathrm{p} \leq 0.05)$.

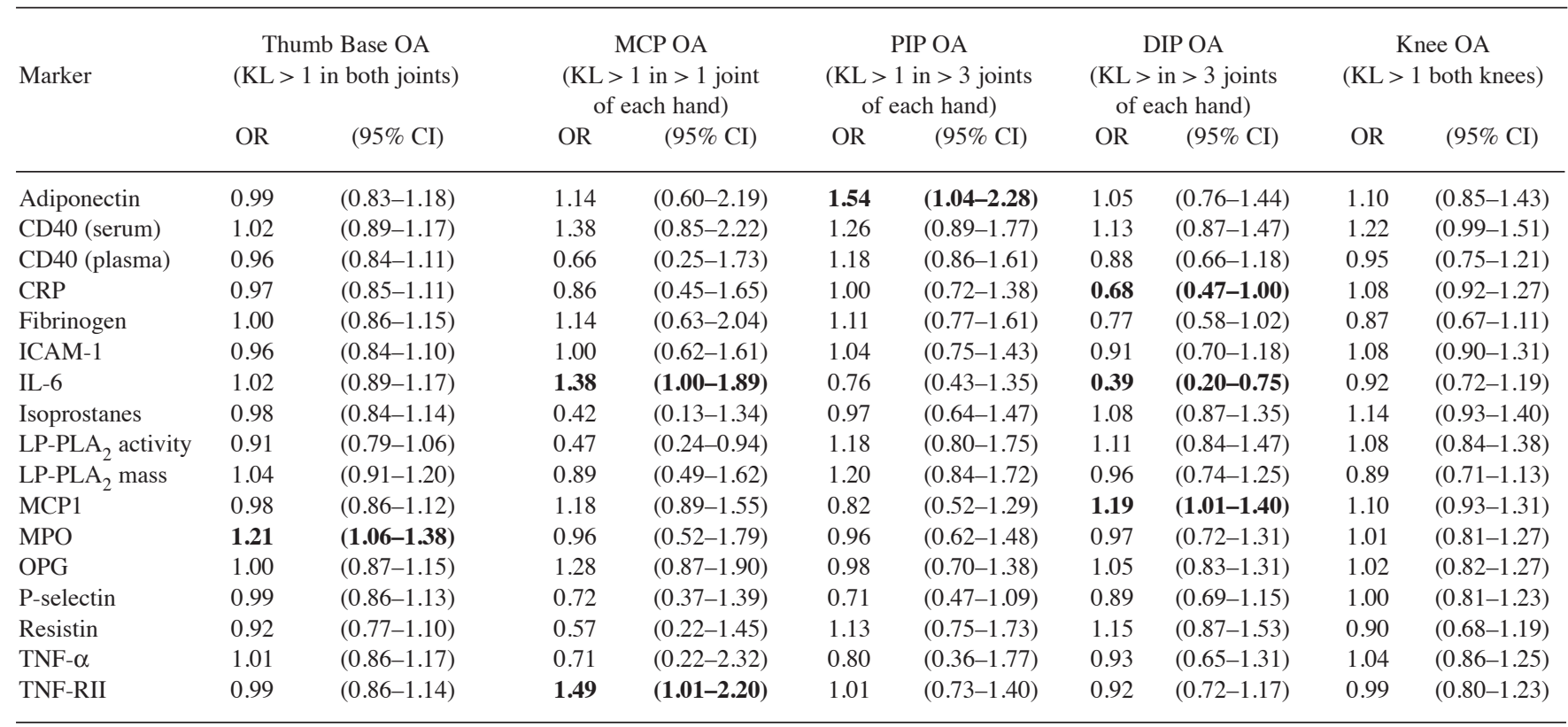

BMI: body mass index; KL: Kellgren and Lawrence grade; CD40: CD40 ligand; CRP: C-reactive protein; ICAM-1:intercellular adhesion molecule-1: IL-6: interleukin 6; isoprostanes: (urinary creatinine)/(8epi-PGF2a); LP-PLA 2 : lipoprotein-associated phospholipase $\mathrm{A}_{2}$; MCP1: monocyte chemoattractant protein; MPO: myeloperoxidase; OPG: osteoprotegerin; TNF- $\alpha$ : tumor necrosis factor- $\alpha$; TNF-RII: type II TNF receptor; PIP: proximal interphalangeal; DIP: distal interphalangeal.

Table 4. Risk of radiographic osteoarthritis (ROA) using number of affected joints as the outcome. Linear regression analysis adjusted for age, sex, and body mass index. Joint score is total number of affected $(\mathrm{KL}>1)$ joints.

\begin{tabular}{|c|c|c|c|c|}
\hline \multirow[t]{2}{*}{ Marker } & \multicolumn{2}{|c|}{$\begin{array}{c}\text { Linear Regression Model } \\
(\text { dependent variable }= \\
\text { joint score })\end{array}$} & \multicolumn{2}{|c|}{$\begin{array}{c}\text { Linear Regression Mode } \\
\text { (dependent variable }= \\
\text { log of joint score })\end{array}$} \\
\hline & $\begin{array}{c}\text { B } \\
\text { Coefficient* }\end{array}$ & $\mathrm{p}$ & $\begin{array}{c}\text { B } \\
\text { Coefficient** }\end{array}$ & $\mathrm{p}$ \\
\hline Adiponectin & -0.028 & 0.86 & 0.005 & 0.88 \\
\hline CD40 (serum) & 0.227 & 0.06 & 0.033 & 0.16 \\
\hline CD40 (plasma) & 0.021 & 0.86 & 0.007 & 0.77 \\
\hline C-reactive protein & -0.053 & 0.67 & -0.004 & 0.88 \\
\hline Fibrinogen & -0.143 & 0.26 & -0.030 & 0.23 \\
\hline ICAM-1 & 0.047 & 0.69 & -0.006 & 0.80 \\
\hline Interleukin 6 & -0.054 & 0.65 & 0.007 & 0.77 \\
\hline Isoprostanes & 0.008 & 0.96 & -0.012 & 0.66 \\
\hline LP-PLA 2 activity & -0.042 & 0.75 & -0.033 & 0.20 \\
\hline LP-PLA 2 mass & -0.086 & 0.47 & -0.025 & 0.28 \\
\hline $\mathrm{MCP} 1$ & 0.085 & 0.48 & 0.012 & 0.61 \\
\hline Myeloperoxidase & 0.149 & 0.21 & 0.037 & 0.11 \\
\hline Osteoprotegerin & 0.114 & 0.38 & 0.009 & 0.73 \\
\hline P-selectin & -0.085 & 0.48 & -0.002 & 0.92 \\
\hline Resistin & -0.026 & 0.86 & -0.024 & 0.41 \\
\hline TNF- $\alpha$ & -0.037 & 0.79 & -0.013 & 0.64 \\
\hline TNF-RII & 0.163 & 0.20 & 0.013 & 0.59 \\
\hline
\end{tabular}

* Change in no. SD needed to predict 1 additional joint affected. ** Change in no. SD needed to predict 1 additional log joint affected. KL: Kellgren and Lawrence grade; CD40: CD40 ligand; ICAM-1: intercellular adhe-

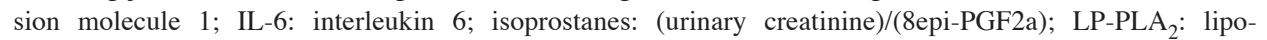
protein-associated phospholipase $\mathrm{A}_{2}$; MCP1: monocyte chemoattractant protein; TNF- $\alpha$ : tumor necrosis factor- $\alpha$; TNF-RII: type II TNF receptor. 
Table 5. Effect of total number of affected joints on log (markers). Linear regression analysis adjusted for age, sex, and body mass index.

\begin{tabular}{|c|c|c|c|}
\hline Marker & $\begin{array}{c}\mathrm{N} \text { in Analysis } \\
\text { (Maximum } \mathrm{n}=1235 \text { ) }\end{array}$ & B Coefficient & $\mathrm{p}$ \\
\hline Adiponectin & 885 & -0.001 & 0.78 \\
\hline CD40 (serum) & 1211 & 0.022 & 0.03 \\
\hline CD40 (plasma) & 1229 & 0.003 & 0.75 \\
\hline C-reactive protein & 1226 & -0.004 & 0.54 \\
\hline Fibrinogen & 1227 & -0.001 & 0.29 \\
\hline ICAM-1 & 1226 & 0.001 & 0.59 \\
\hline Interleukin 6 & 1225 & -0.005 & 0.22 \\
\hline Isoprostanes & 964 & 0.003 & 0.52 \\
\hline LP-PLA ${ }_{2}$ activity & 1227 & -0.001 & 0.74 \\
\hline LP-PLA $_{2}$ mass & 1227 & -0.001 & 0.67 \\
\hline MCP1 & 1208 & 0.000 & 0.97 \\
\hline Myeloperoxidase & 1182 & 0.007 & 0.10 \\
\hline Osteoprotegerin & 1227 & 0.001 & 0.66 \\
\hline P-selectin & 1229 & 0.003 & 0.27 \\
\hline Resistin & 876 & 0.000 & 0.97 \\
\hline TNF- $\alpha$ & 877 & 0.000 & 0.90 \\
\hline TNF-RII & 1194 & 0.003 & 0.12 \\
\hline
\end{tabular}

CD40: CD40 ligand; ICAM-1: intercellular adhesion molecule 1; isoprostanes: (urinary creatinine)/(8epi-PGF2a); LP-PLA 2 : lipoprotein-associated phospholipase $\mathrm{A}_{2}$; $\mathrm{MCP} 1$ : monocyte chemoattractant protein; TNF- $\alpha$ : tumor necrosis factor- $\alpha$; TNF-RII: type II TNF receptor.

observe this effect. We would argue that these 2 studies suggest that while CRP genotype could be associated with the development of OA, this relationship is highly confounded by the relationship between CRP and body mass. This would make CRP a poor biomarker for the development of OA in clinical situations.

That we were unable to find a consistent relationship between inflammatory markers and OA after adjustment for BMI, which has a relatively weak association with systemic inflammation, suggests that the inclusion of a measure of body habitus with a stronger relationship to inflammation, such as waist circumference, would not have changed our results.

Regarding the relationship between other inflammatory markers and OA, there are a number of reasons why our findings might not agree with other reported associations. First, it is possible that certain OA phenotypes are more likely to be associated with higher levels of inflammation and that these phenotypes are more likely to be identified using blood-derived markers. Studies of rapidly progressive hip $\mathrm{OA}^{7}$ or "erosive" hand $\mathrm{OA}^{8,18}$, for example, may reflect discrete OA subtypes in which local inflammation plays an especially important role and is significant enough to be reflected in the serum.

Second, some studies have suggested that clinically detected OA-associated synovitis ${ }^{15}$, or synovitis detected in pathologic specimens ${ }^{9}$, are more likely to be associated with painful OA phenotypes, and that these phenotypes may in turn be more highly associated with markers of systemic inflammation. Another study ${ }^{10}$ found that CRP was more associated with degree of pain in OA rather than radiographic disease, further supporting this hypothesis. Our sensitivity analysis did not find any association between CRP and symptomatic OA (although comparatively strong associations between some definitions of symptomatic OA and fibrinogen, IL-6, and LP-PLA ${ }_{2}$ might warrant further investigation).

Third, publication bias could be a source of previously reported associations. For instance, we identified some associations that we believe are due to chance. Some of these associations were weak (e.g., MCP-1, myeloperoxidase), while others gave opposite effects depending on OA phenotype (a negative association between IL-6 and PIP joint OA, but a positive association with $\mathrm{MCP}$ joint $\mathrm{OA}$ ), making their interpretation problematic. Stronger associations (e.g., TNF-RII and adiponectin) could reflect chance associations given that we investigated 17 different markers in multiple models. It is possible that other authors published their more interesting results, while less interesting or negative associations were not published. It is intriguing, however, that some of the associations we found have been reported by other authors including those for TNF-RII ${ }^{14}$, IL- $6^{14,15}$, and adiponectin ${ }^{18}$ (the latter, interestingly, in a similar phenotype of hand OA). Further research into these markers in appropriate datasets might be of value to help clarify whether these relationships are due to chance or are true.

Fourth, timing may play an important role. In most studies, the inflammatory marker was tested in patients with active OA. This may not have been the case in our study, in which blood was drawn some years before radiographs were taken; some patients may not have had OA yet or may have been developing $\mathrm{OA}$ at the time of the blood draw. On the other hand, at least 1 study has suggested that a rise in CRP may precede radiographic disease by some years ${ }^{29}$; thus it could be preferable to have blood drawn prior to disease onset. Therefore, it is difficult to be sure how to interpret this apparent limitation.

Finally, it is possible that differences with other published results reflect the limitations of our cross-sectional study design. We did not, for example, evaluate changes in inflammatory marker levels or in the degree of ROA over time. For example, 1 study found that elevated CRP levels were associated with more rapid progression of disease ${ }^{5}$. Our study did not examine changes in markers or joints over time.

We have shown that in general, serum inflammatory markers are poorly associated cross-sectionally with radiographic arthritis of the hands and/or knees. A few markers, including IL-6, adiponectin, LP-PLA 2 , and TNF-RII, may warrant further investigation as markers for some OA phenotypes. Additionally, further study is needed to establish whether any of these markers would perform better as markers of more aggressive disease.

\section{REFERENCES}

1. Bonnet CS, Walsh DA. Osteoarthritis, angiogenesis and inflammation. Rheumatology 2005;44:7-16.

2. Aigner T, Van der Kraan P, Van den Berg W. Osteoarthritis and

Personal non-commercial use only. The Journal of Rheumatology Copyright @ 2011. All rights reserved. 
inflammation - inflammatory changes in osteoarthritic synoviopathy. In: Buckwalter JA, Lotz M, Stoltz J-F, editors. Osteoarthritis, inflammation and degradation: a continuum. Amsterdam: IOS Press; 2007:219-35.

3. Pelletier JP, Martel-Pelletier J, Abramson SB. Osteoarthritis, an inflammatory disease: potential implication for the selection of new therapeutic targets. Arthritis Rheum 2001;44:1237-47.

4. Sharif M, Elson CJ, Dieppe PA, Kirwan JR. Elevated serum C-reactive protein levels in osteoarthritis. Br J Rheumatol 1997;36:140-1.

5. Spector TD, Hart DJ, Nandra D, Doyle DV, Mackillop N, Gallimore JR, et al. Low-level increases in serum C-reactive protein are present in early osteoarthritis of the knee and predict progressive disease. Arthritis Rheum 1997;40:723-7.

6. Wolfe F. The C-reactive protein but not erythrocyte sedimentation rate is associated with clinical severity in patients with osteoarthritis of the knee or hip. J Rheumatol 1997;24:1486-8.

7. Conrozier T, Carlier MC, Mathieu P, Colson F, Debard AL, Richard $\mathrm{S}$, et al. Serum levels of YKL-40 and C reactive protein in patients with hip osteoarthritis and healthy subjects: a cross sectional study. Ann Rheum Dis 2000;59:828-31.

8. Punzi L, Ramonda R, Oliviero F, Sfriso P, Mussap M, Plebani M, et al. Value of $C$ reactive protein in the assessment of erosive osteoarthritis of the hand. Ann Rheum Dis 2005;64:955-7.

9. Pearle AD, Scanzello CR, George S, Mandl LA, DiCarlo EF, Peterson M, et al. Elevated high-sensitivity C-reactive protein levels are associated with local inflammatory findings in patients with osteoarthritis. Osteoarthritis Cartilage 2007;15:516-23.

10. Sturmer T, Brenner H, Koenig W, Gunther KP. Severity and extent of osteoarthritis and low grade systemic inflammation as assessed by high sensitivity $\mathrm{C}$ reactive protein. Ann Rheum Dis 2004;63:200-5

11. Davis CR, Karl J, Granell R, Kirwan JR, Fasham J, Johansen J, et al. Can biochemical markers serve as surrogates for imaging in knee osteoarthritis? Arthritis Rheum 2007;56:4038-47.

12. Kraus VB, Stabler TV, Luta G, Renner JB, Dragomir AD, Jordan JM. Interpretation of serum C-reactive protein (CRP) levels for cardiovascular disease risk is complicated by race, pulmonary disease, body mass index, gender, and osteoarthritis. Osteoarthritis Cartilage 2007;15:966-71.

13. Nevitt M, Felson D, Peterfy C, Wildy K, Ling S, Land L, et al. Inflammation markers (CRP, TNF-alpha, IL-6) are not associated with radiographic or MRI findings of knee OA in the elderly: The Health ABC Study [abstract]. Arthritis Rheum 2002;46 Suppl:S372-3.

14. Otterness IG, Swindell AC, Zimmerer RO, Poole AR, Ionescu M, Weiner E. An analysis of 14 molecular markers for monitoring osteoarthritis: segregation of the markers into clusters and distinguishing osteoarthritis at baseline. Osteoarthritis Cartilage 2000;8:180-5.

15. Toncheva A, Remichkova M, Ikonomova K, Dimitrova P, Ivanovska N. Inflammatory response in patients with active and inactive osteoarthritis. Rheumatol Int 2009;29:1197-203.

16. Fraenkel L, Roubenoff R, LaValley M, McAlindon T, Chaisson C, Evans $\mathrm{S}$, et al. The association of peripheral monocyte derived interleukin 1 beta (IL-1 beta), IL-1 receptor antagonist, and tumor necrosis factor-alpha with osteoarthritis in the elderly. J Rheumatol 1998;25:1820-6.
17. Lavigne P, Benderdour M, Lajeunesse D, Shi Q, Fernandes JC. Expression of ICAM-1 by osteoblasts in healthy individuals and in patients suffering from osteoarthritis and osteoporosis. Bone 2004;35:463-70.

18. Filková M, Lisková M, Hulejová H, Haluzík M, Gatterová J, Pavelková A, et al. Increased serum adiponectin levels in female patients with erosive compared with non-erosive osteoarthritis. Ann Rheum Dis 2009;68:295-6.

19. Kannel WB, Feinleib M, McNamara PM, Garrison RJ, Castelli WP. An investigation of coronary heart disease in families: the Framingham Offspring Study. Am J Epidemiol 1979;110:281.

20. Schnabel R, Larson MG, Dupuis J, Lunetta KL, Lipinska I, Meigs $\mathrm{JB}$, et al. Relations of inflammatory biomarkers and common genetic variants with arterial stiffness and wave reflection. Hypertension 2008;51:1651-7.

21. Felson DT, Zhang Y, Hannan MT, Naimark A, Weissman B, Aliabadi $\mathrm{P}$, et al. Risk factors for incident radiographic knee osteoarthritis in the elderly: the Framingham Study. Arthritis Rheum 1997;40:728-33.

22. Zhang Y, Glynn RJ, Felson DT. Musculoskeletal disease research: should we analyze the joint or the person? J Rheumatol 1996;23:1130-4.

23. Kellgren JH, Lawrence JS. Radiological assessment of osteoarthrosis. Ann Rheum Dis 1957;16:494-501.

24. Kerkhof HJ, Bierma-Zeinstra SM, Castano-Betancourt MC, de Maat MP, Hofman A, Pols HA, et al. Serum C reactive protein levels and genetic variation in the CRP gene are not associated with the prevalence, incidence or progression of osteoarthritis independent of body mass index. Ann Rheum Dis 2010;69:1976-82.

25. Garnero P, Mazières B, Guéguen A, Abbal M, Berdah L, Lequesne $\mathrm{M}$, et al. Cross-sectional association of 10 molecular markers of bone, cartilage, and synovium with disease activity and radiological joint damage in patients with hip osteoarthritis: the ECHODIAH cohort. J Rheumatol 2005;32:697-703

26. Takahashi M, Naito K, Abe M, Sawada T, Nagano A. Relationship between radiographic grading of osteoarthritis and the biochemical markers for arthritis in knee osteoarthritis. Arthritis Res Ther 2004;6:R208-12.

27. Sowers M, Jannausch M, Stein E, Jamadar D, Hochberg M, Lachance L. C-reactive protein as a biomarker of emergent osteoarthritis. Osteoarthritis Cartilage 2002;10:595-601.

28. Bos SD, Suchiman HE, Kloppenburg M, Houwing-Duistermaat JJ, le Graverand MP, Seymour AB, et al. Allelic variation at the $\mathrm{C}$-reactive protein gene associates to both hand osteoarthritis severity and serum high sensitive C-reactive protein levels in the GARP study. Ann Rheum Dis 2008;67:877-9.

29. Sharif M, Shepstone L, Elson CJ, Dieppe PA, Kirwan JR. Increased serum $\mathrm{C}$ reactive protein may reflect events that precede radiographic progression in osteoarthritis of the knee. Ann Rheum Dis 2000;59:71-4. 